\title{
CASO 10-2014: ADULTO MAYOR GON ENFERMEDAD PULMONAR INTERSTICIAL DIFUSA
}

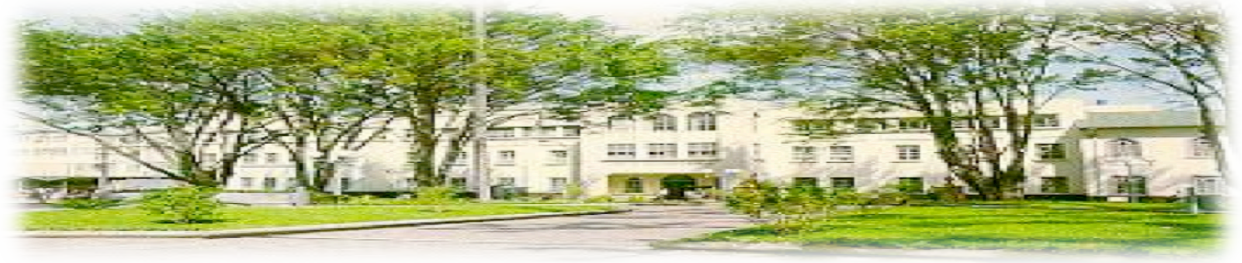

Hospital San quan de Dios. San José. Costa Rica. Fundado en 1845

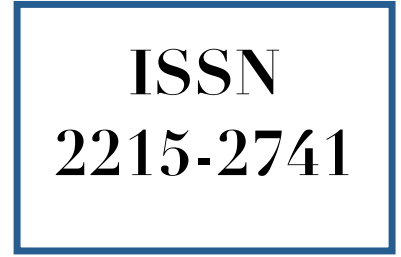

\section{Abordaje Diagnóstico}

$\begin{array}{ll}\text { Recibido: } & 25 / 09 / 2013 \\ \text { Aceptado: } & 26 / 03 / 2014\end{array}$

Pablo Coste Murillo

${ }^{\mathbf{1}}$ Médico Residente de Medicina Interna. Programa de Estudios de Postgrado UCR- CENDEISSS. Hospital San Juan de Dios. Correo electrónico: costepablo@gmail.com

\section{RESUMEN}

Las enfermedades pulmonares intersticiales difusas constituyen un grupo diverso de más de 200 entidades clínicas, caracterizadas por grados variables de fibrosis e inflamación del parénquima e intersticio pulmonar ${ }^{(1)}$. Dada la amplia y heterogénea gama de enfermedades que se incluyen bajo este término, es indispensable un abordaje ordenado y completo que permita establecer un diagnóstico específico y un tratamiento adecuado. Este punto tiende a ser difícil para el médico tratante, quien frecuentemente carece de un ordenamiento diagnóstico adecuado. El presente artículo ofrece un caso clínico ilustrativo y una guía para el abordaje diagnóstico del paciente con sospecha de EPID, basado en el Consenso Multidisciplinario Internacional de la Sociedad Torácica Americana (ATS) y la Sociedad Respiratoria Europea (ERS) ${ }^{(2)}$.

\section{PALABRAS CLAVE}

Enfermedad pulmonar intersticial difusa. Neumonía intersticial idiopática. fibrosis pulmonar idiopática. Neumonitis intersticial usual. Neumonía intersticial no específica. Neumonía intersticial inclasificable. Neumonitis por hipersensibilidad.

\section{ABSTRACT}

Diffuse interstitial lung diseases are a diverse group of more than 200 clinical entities characterized by varying degrees of fibrosis and inflammation of the lung parenchyma and interstitium. Given the wide and diverse range of diseases included under this term, it is essential to have a complete and orderly approach to establish a specific diagnosis and appropriate treatment. This issue tends to be difficult for the physician 
who often lacks a proper diagnosis ordering. This article provides a case illustration and a guide to the diagnostic approach of patients suspected of having ILD-based International Multidisciplinary Consensus American Thoracic Society (ATS) and European Respiratory Society $(\mathrm{ERS})^{(2)}$.

\section{KEY WORDS}

Diffuse parenchymal lung diseases. Idiopathic interstitial pneumonia. Idiopathic pulmonary fibrosis. Usual interstitial pneumonia. Nonspecific interstitial pneumonia. Unclassifiable interstitial pneumonia. Hypersensitivity pneumonitis.

\section{CASO CLÍNICO}

Femenina de 79 años, trabajadora de textilera por 30 años, no tabaquista, con antecedente de lumbalgia postural crónica, insuficiencia venosa crónica y enfermedad pulmonar intersticial difusa (EPID).

Valorada en consulta externa de neumología en agosto de 2002 por cuadro crónico de 6 meses de tos productiva y expectoración amarillenta. El examen físico (EF) mostró escasas crepitaciones bibasales y ausencia de hipocratismo digital. Las pruebas de función respiratoria (PFR) fueron normales. $\mathrm{La}$ tomografía computarizada (TC) reportó un infiltrado pulmonar reticular grueso bilateral compatible con intersticio pulmonar peribroncovascular engrosado y el ecocardiograma (ECO) evidenció insuficiencia mitral leve y aortoesclerosis, sin datos de hipertensión pulmonar. Se programó broncoscopía.

La paciente perdió el control médico hasta noviembre de 2011, se desconoce el motivo. En ese momento reconsultó por cuadro de un año de disnea de pequeños esfuerzos y tos seca. Al EF CsPs con MV disminuido, crépitos secos difusos bilaterales asociados a sibilancias. Se documentó hipocratismo digital.

Se realizó una broncoscopía que mostró cambios inespecíficos inflamatorios y los cultivos demostraron escaso crecimiento bacteriano, siendo negativos por hongos, M. tuberculosis y otras micobacterias. La citología reportó cambios inflamatorios sin atipia celular y el lavado bronquioloalveolar (LBA) evidenció un diferencial con 56\% macrófagos, 11\% PMN y $1 \%$ eosinófílos.

La TC demostró pequeñas adenopatías mediastinales y pretraqueales, múltiples adenomegalias densamente calcificadas, engrosamiento de los septos intersticiales de predominio en lóbulos inferiores y un patrón periférico, peribroncovascular y heterogéneo con escasa panalización bibasal. Aunaba abundante vidrio esmerilado y escasas bronquiectasias cilíndricas por tracción.

El ECO reportó hipertrofia ventricular izquierda moderada concéntrica, dilatación moderada del ventrículo derecho y leve biauricular, insuficiencia tricuspídea y pulmonar leve, con hipertensión arterial pulmonar severa y disfunción diastólica tipo 1.

Entre otros exámenes, la serie gastroduodenal puso de manifiesto un reflujo esófago - esófago y en la caminata de 6 min se reportó un ritmo de marcha lento, con un $87 \%$ de oxigenación basal y un $67 \%$ a los tres minutos, con una distancia recorrida de $210 \mathrm{~m}$ y ameritó la suspensión de la misma, debido a disnea progresiva.

Las PFR evidenciaron un patrón restrictivo, con capacidades y volúmenes en disminución progresiva $y$ acelerada pese a tratamiento desescalonado con prednisona durante un año y ocho meses. Las pruebas de difusión de $\mathrm{CO}_{2}$ mostraron el mismo patrón.

En diciembre de 2012, la paciente consultó por cuadro agudo de malestar general, sensación febril, tos productiva y disnea exacerbada. Al EF cursaba hipotensa, taquipneica, desaturada, febril y con franca dificultad respiratoria. Se diagnosticó choque séptico secundario a bronconeumonía adquirida en la comunidad. Se inició ventilación mecánica, soporte inotrópico, esteroides y antibioticoterapia.

La paciente fue hospitalizada en una unidad de cuidados críticos, evolucionó tórpidamente $\mathrm{y}$ falleció al octavo día de internamiento. El estudio patológico post morten a nivel pulmonar 
evidenció fibrosis pulmonar secundaria a una neumonitis intersticial con patrón usual.

\section{DISCUSIÓN}

Bajo el término de EPID se incluyen más de 200 entidades diferentes que se caracterizan por cuatro manifestaciones principales ${ }^{(2)}$.

1. Síntomas respiratorios como disnea y tos.

2. Anormalidades radiográficas específicas.

3. Pruebas de función pulmonar con volúmenes pulmonares disminuídos y

4. Un patrón histológico con inflamación y fibrosis.

Las EPID se dividen en: Enfermedades con causa conocida (asociadas a enfermedades del tejido conectivo, exposiciones ambientales o a medicamentos) y con causa desconocida, dentro de las que se incluyen las Neumonías intersticiales idiopáticas (NII), las Enfermedades pulmonares granulomatosas como la sarcoidosis y Otras enfermedades, que incluyen la linfangioleiomiomatosis y la histiocitosis $\mathrm{X}^{(2)}$.

Las diversas características clínicas, radiológicas e histológicas permite clasificar a las NII en siete entidades distintas, las cuales se enumeran de acuerdo a su incidencia ${ }^{(2)}$ :

1. Fibrosis pulmonar idiopática (FPI)

2. Neumonía intersticial no específica (NINE)

3. Neumonía criptogénica organizativa (NCO)

4. Neumonía intersticial aguda (NIA)

5. Enfermedad interticial difusa asociada a bronquiolitis respiratoria (EPID- BR)

6. Neumonía intersticial descamativa (NID) y

7. Neumonía intersticial linfoidea (NIL)

Debido a la amplia cantidad de patologías distintas que se incluyen dentro del término de EPID, se ha concluido que el diagnóstico debe hacerse una vez que haya sido valorada toda la evidencia clínica, radiológica e histológica disponible $^{(2)}$. Por este motivo, el médico tratante del paciente con EPID debe estar acostumbrado a realizar un adecuado y ordenado trabajo diagnóstico, con base en los elementos disponibles.
El proceso diagnóstico inicia con una evaluación primaria que implica una historia clínica completa, un examen físico minucioso, una radiografía de tórax y pruebas de función pulmonar $^{(2)}$. El propósito fundamental en este momento es diferenciar aquellas EPID asociadas a enfermedades con una causa identificable de las que impresionan ser una posible $\mathrm{NII}^{(2)}$.

Si al finalizar este abordaje no se identificó una causa del cuadro, la tomografía computarizada de alta resolución (TCAR) debe pasar a formar parte de la evaluación integral ${ }^{(2)}$. Su papel principal consiste en separar a los pacientes con rasgos radiológicos de Fibrosis Pulmonar Idiopática (FPI) de aquellos con rasgos menos específicos asociados a otras $\mathrm{NII}^{(2)}$.

En consenso internacional y con base en distintas características imagenológicas valorables en una TCAR, se han establecido tres patrones tomográficas para FPI: definitivo, posible e inconsistente $^{(3)}$. Varios estudios han documentado un valor predictivo positivo del 90 al $100 \%$ para el diagnóstico de Neumonía Intersticial Usual (NIU) con base en estos criterios $^{(3)}$.

Para establecer el diagnóstico tomográfico definitivo de NIU, debe haber cuatro características presentes:

- Infiltrados reticulares.

- Predominio subpleural y basal.

- Panal de abeja con o sin bronquiectasias por tracción.

- Ausencia de cualquiera de las siete características catalogadas como inconsistente para $\mathrm{NIU}^{(3)}$ :

1. Predominio superior/medio.

2. Predominio peribroncovascular.

3. Vidrio esmerilado extenso.

4. Micronódulos profusos.

5. Quistes discretos.

6. Mosaico atenuado difuso.

7. Consolidación.

Si los hallazgos tomográficos no satisfacen los criterios de Definitivo para NIU, es necesaria la realización de una biopsia quirúrgica para orientar el diagnóstico ${ }^{(2)}$. Ésta debe realizarse de más de un lóbulo, debido a la posibilidad de que 
una única muestra sea poco concluyente o que incluso presente más de un patrón histológico determinado $^{(4)}$. En este último caso debe diagnosticarse la enfermedad que presente una evolución clínica y radiológica más compatible o tenga hallazgos histológicos más específicos ${ }^{(2)}$. En cuanto a la biopsia transbronquial, su rol primordial en el estudio de EPID consiste en excluir causas específicas como sarcoidosis, neoplasias y ciertas infecciones ${ }^{(2)}$.

Entre otros exámenes disponibles se encuentra el lavado broquioalveolar (LBA), el cual no es un examen diagnóstico en el estudio de las EPID $^{(2)}$. $\mathrm{Su}$ utilidad principal radica en excluir causas infecciosas $o$ tumores $y$ pese a no ser diagnóstico, un patrón celular típico puede contribuir al abordaje diferencial en casos difíciles y específicos, por ejemplo, en proteinosis alveolar y en la histiocitosis de Langerhans ${ }^{(2)}$. Asimismo, debe tomarse en cuenta que un diferencial normal en el LBA no excluye la presencia de anormalidades microscópicas en el tejido pulmonar ${ }^{(5)}$.

Una vez que se ha concluido el estudio diagnóstico del paciente con EPID (clínico, radiológico e histológico), un pequeño porcentaje de pacientes permanece sin diagnóstico, debido a hallazgos inconclusos o contradictorios $^{(2)}$. Para estos casos y al igual que en otros sistemas de clasificación, en el consenso multidisciplinario internacional de 2002 para la clasificación de las NII de la Sociedad Torácica Americana y la Sociedad Respiratoria Europea (STA/SRE) se incluyó una categoría denominada Neumonía Intersticial Inclasificable. Aquí se incluyen casos que no pueden ser clasificados por varias razones: información clínica o radiológica inadecuada, biopsia inadecuada, discrepancias mayores en los hallazgos clínico, radiológicos e histológicos y/o discrepancias mayores en los hallazgos histológicas en múltiples biopsias del mismo caso ${ }^{(2)}$.

En el presente caso, se abordó inicialmente desde el plano clínico y radiológico. El caso se trata de una adulta mayor, trabajadora textil, no tabaquista, con cuadro crónico de disnea progresiva y tos seca, con mala respuesta a esteroides. Al examen físico con crepitaciones secas, difusas y bilaterales; con acropaquias. La TC no fue de alta resolución y reportó adenopatías calcificadas, engrosamiento intersticial del lóbulo inferior y medio, patrón periférico peribroncovascular heterogéneo, panalización bibasal escasa, bronquiectasias por tracción y abundante vidrio esmerilado.

Entre otros exámenes, las pruebas de función respiratoria con un patrón restrictivo progresivo, una $\mathrm{DLCO}_{2}$ disminuída al menos del $50 \%$, un Ecocardiograma con hipertensión pulmonar severa, una serie gastroduodenal con reflujo esófago - esófago y un LBA con marcada linfocitosis.

Con base en al abordaje diagnóstico de EPID ya descrito, con la información clínica y radiológica disponible no es posible clasificarla como una EPID con causa identificable, ante la ausencia de una historia clínica completa y un seguimiento cercano. Al complementar con los hallazgos de la TC se puede descartar la FPI, ya que presenta varias características tomográficas inconsistentes para $\mathrm{NIU}^{(3)}$.

Mediante un abordaje etiopatogénico con abanico de posibilidades ${ }^{(6)}$, tomando en cuenta la información clínica y radiológica disponible y al tratarse de una EPID de evolución crónica en una paciente adulta mayor, con lumbalgia crónica, no fumadora, trabajadora textil y con los hallazgos tomográficos descritos, se determinó que el diagnóstico diferencial principal incluia una EPID asociada a espondilitis anquilosante (EA), una neumonía intersticial no específica y una neumonitis por hipersensibilidad asociada al pulmón del textilero.

Diversas características hacen la presencia de una EA menos probable en comparación con las otras posibilidades. Algunas de ellas incluyen la ausencia de una afección articular más característica, es más frecuente en varones de 30 a 40 años, las manifestaciones pulmonares principales incluyen la restricción anquilosante de la pared torácica, la enfermedad fibrobulosa apical, el neumotórax espontáneo y la sobreinfección pulmonar ${ }^{(7)}$.

En cuanto a la NINE, ésta suele ser más frecuente en mujeres de 26 a 73 años, no fumadoras, los síntomas más frecuentes son tos y disnea, la supervivencia a 10 años es de $73.2 \%$ y tomográficamente suele evidenciar distribución 
predominantemente inferior y periférica o difusa, con bronquiectasias por tracción, reticulación, pérdida de volumen y vidrio esmerilado hasta en un $44 \%{ }^{(8)}$. Desde el punto de vista clínico y radiológico, esta entidad es bastante compatible con nuestro caso.

La NH crónica inicia de forma insidiosa con tos, disnea, pérdida de peso y fatiga. Las crepitaciones bibasales usualmente están presentes $^{(9)}$. La TCAR evidencia múltiples patrones. Comúnmente hay nódulos centrolobulares múltiples de 2 a $4 \mathrm{~mm}$, difusos, con algunas áreas de vidrio esmerilado fundamentalmente en lóbulos inferiores, hallazgos que suelen resolverse al detener la exposición al antígeno. Es muy frecuente que los hallazgos en la TCAR asemejen los de una $\mathrm{FPI}^{(1)}$. En nuestro caso, al no haber una historia clínica completa, se desconoce si hubo exposición a algún antígeno desencadenante, asimismo al no haber un antígeno identificado, tampoco hay evidencia de anticuerpos precipitantes positivos. Los síntomas son compatibles y los hallazgos tomográficos no descartan la NH. La probabilidad de que se trate de esta entidad no es despreciable.

En este punto y al haber analizado el caso desde la perspectiva clínica y radiológica, las principales opciones diagnósticas son la NSIP y la $\mathrm{NH}$ crónica. Al continuar con el estudio diagnóstico de las EPID, el siguiente paso al no lograr determinar una causa específica de $\mathrm{NH}$ y al existir la posibilidad de una NSIP, consiste en la realización de una biopsia y su estudio histológico $^{(3)}$.

En el reporte patológico de pulmón describió: "en pleura se observa leve edema pero sin producir engrosamiento de la misma. Se identifican zonas afectadas alternando con zonas de parénquima pulmonar normal o menos comprometido" (Fig1).

En alto poder en las zonas afectadas se observan zonas de engrosamiento de las paredes alveolares a expensas de fibrosis y congestión. Con tinciones especiales de tricrómico y retículo se identifican algunos tabiques con escasos fibroblastos y otros con un mayor depósito de colágeno e inclusive fibras elásticas, lo que nos habla de que existe una heterogeneidad temporal en este proceso de reparación (Fig 2), lo cual es más compatible con una NIU.

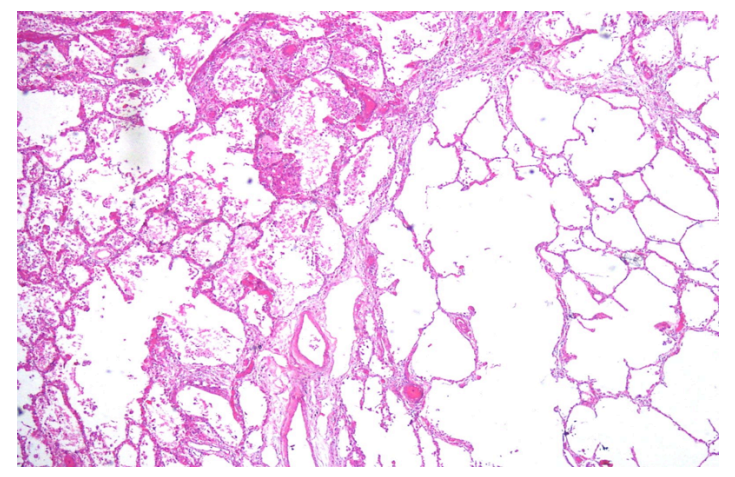

Figura 1. Histología pulmonar a bajo poder. Fuente: Servicio de Patología HSJD.

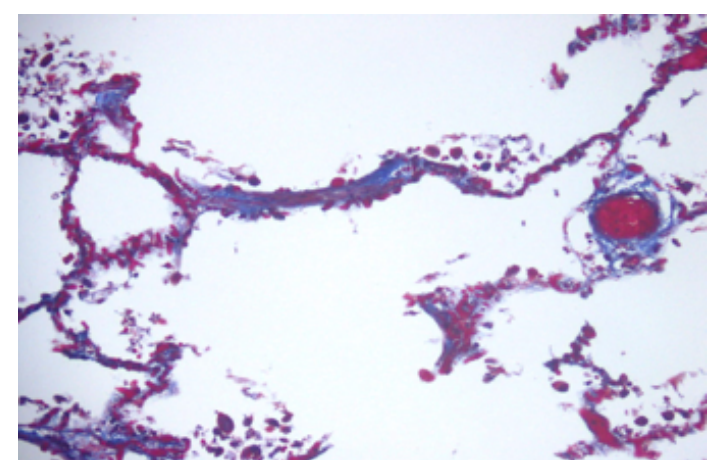

Figura 2. Histología pulmonar a alto poder

Fuente: Servicio de Patología HSJD.

Con base en esta información y de acuerdo al consenso multidisciplinario internacional de 2002 para la clasificación de las NII de la ATS/ERS, el patrón de NIU con baja magnificación es heterogéneo, alternando zonas de fibrosis y panal de abeja con pulmón normal o poco afectado, con predominio subpleural y paraseptal $^{(3)}$. La inflamación es leve y la presencia de seis características descartan este patrón: membranas hialinas, neumonía organizativa, granulomas, infiltrado intersticial inflamatorio abudante, cambios predominantemente centrales y presencia de otras características sugestivas de otras entidades $^{(10)}$.

Con estas características se estableció un sistema de clasificación para convenir el nivel de confianza para un diagnóstico histopatólógico de 
NIU. Diversas características establecen el diagnóstico histológico de NIU como definitivo, probable, posible y no $\mathrm{NIU}^{(10)}$. De acuerdo con este sistema de clasificación y al compararlo con los hallazgos reportados en el informe histológico del presente caso, el patrón corresponde a una posible $\mathrm{NIU}^{(10)}$.

La importancia de definir esto radica en que si bien el patrón histológico de NIU es más frecuente en FPI, también puede encontrarse en neumonías intersticiales asociadas a enfermedades del tejido conectivo y neumonitis por hipersensibilidad ${ }^{(3)}$. Por esta razón, en el presente caso, al ser estas dos posibilidades diagnósticas, es importante definir que el patrón corresponde a una posible NIU.

Por otro lado, el patrón histológico de NINE se caracteriza por una relativa homogeneidad espacial y temporal de la lesión inflamatoria y fibrótica, características ausentes en la histología de nuestro caso $^{(10)}$.

En resumen, en este caso la clínica y radiología no descartan ni confirman la presencia de una EPID asociada a una enfermedad del tejido conectivo o a neumonitis por hipersensibilidad, siendo más fuerte la segunda posibilidad. Cuando aunamos la histología, la misma ayuda a descartar la posibilidad de una NINE, pero no logra dilucidar una etiología específica. Esta información permite concluir que corresponde a una NIU, pero al ser una TC inconsistente para FPI/NIU y una histología de posible FPI/NIU, se puede descartar la presencia de $\mathrm{FPI}^{(3)}$.

Al basarse en el consenso multidisciplinario internacional de 2002 para la clasificación de las NII de la ATS/ERS, al existir una información clínica incompleta, una tomografía axial sin alta resolución, una biopsia post mortem en un paciente previamente tratado con esteroides y discrepancias clínico, radiológicos e histológicas, se puede concluir que nuestro caso ejemplifica una Neumonía Intersticial Inclasificable.

\section{CONCLUSIONES}

En el estudio de pacientes con EPID, debe tenerse presente que el alcanzar el diagnóstico definitivo es un proceso dinámico que exige valorar la evolución del paciente y el diagnóstico final debe establecerse al valorar en conjunto la clínica, radiología e histología disponible.

En el proceso diagnóstico, el abordaje inicial debe dirigirse a encontrar una EPID con causa conocida. De no evidenciarse una, debe realizarse una TC de alta resolución con el fin de separar a los pacientes con hallazgos típicos de FPI de los asociados a otras NII. En este proceso, el LBA es una herramienta que refuerza una sospecha etiológica y sólo en casos específicos brinda un adecuado valor diagnóstico.

Si la duda diagnóstica persiste, la biopsia quirúrgica de varios lóbulos está indicada en pacientes con EPID que no presentan una presentación clínica $\mathrm{y} / \mathrm{o}$ radiológica típica de FPI. La transbronquial es poco útil y su importancia principal radica en excluir sarcoidosis y ciertas infecciones.

Si completado este abordaje terapéutico no se puede establecer un diagnóstico certero, debe establecerse el diagnóstico de Neumonía Intersticial No Clasificable, sin embargo este es un recurso que debe minimizarse para casos específicos. Mientras tanto deben desestimarse los esquemas terapéuticos hasta concertar un esfuerzo para establecer un diagnóstico firme y excluir causas infecciosas y tumorales.

\section{BIBLIOGRAFÍA}

1. Fishman A Elias J Fishman J Grippi M Senior R Pack A. Fishman's Pumonary Diseases and Disorders. Fourth Edition, Vol 1, Chapter 69, 1161- 1172.

2. American Thoracic Society, European Respiratory Society. American Thoracic Society/European Respiratory Society International Multidisciplinary Consensus Classification of the Idiopathic Interstitial Pneumonias. Am J Respir Crit Care Med. 2002;165: 277-304.

3. G Raghu H Collard J Egan et al. An Official ATS/ERS/JRS/ALAT Statement: Idiopathic Pulmonary Fibrosis: Evidence-based Guidelines for Diagnosis and Management. Am J Respir Crit Care Med. 2011;183:788-824.

4. Flaherty KR Travis WD Colby TV et al. Histological variability in patients with sus- 
pected idiopathic pulmonary fibrosis. Am J Respir Crit Care Med. 2001;164:1722-1727.

5. K Meyer G Raghu R Baughman et al. An Official American Thoracic Society Clinical Practice Guideline: The Clinical Utility of Bronchoalveolar Lavage Cellular Analysis in Interstitial Lung Disease. Am J Respir Crit Care Med. 2012;185(9):1004-1014.

6. E castro L F Briceño. Metodología del Análisis Clínico. Un enfoque didáctico en la enseñanza y la práctica de la medicina. San José. C.R. EDNASSS. 2007.

7. Kanathur N Lee-Chiong T. Pulmonary Manifestations of Ankylosing Spondylitis. Clin Chest Med. 2010;31:547-554.

8. Travis W Hunninghake $\mathrm{G}$ King $\mathrm{T}$ et al. Idiopathic Nonspecific Interstitial Pneumonia Report of an American Thoracic Society Project. Am J Respir Crit Care Med. 2008;177:1338-1347.

9. Lacasse Y Selman M Costabel U et al. Clinical Diagnosis of Hypersensitivity Pneumonitis. American Journal of Respiratory and Critical Care Medicine. 2003;168:952-958.

10. Larsen B Colby T. Update for Pathologists on Idiopathic Interstitial Pneumonias. Arch Pathol Lab Med. 2012;136(10):1234-1241.

\section{CONFLICTO DE INTERÉS}

Los autores declaran que no existió ningún conflicto de interés en el presente reporte.

\section{AGRADECIMIENTO}

Al Servicio de Patología del Hospital San Juan de Dios por las láminas histológicas del paciente. 